\title{
Functionalized $\mathrm{Cd}_{0.5} \mathrm{Zn}_{0.5} \mathrm{~S}$ Chalcogenide Nanotwins Enabling Z- Scheme Photocatalytic Water Splitting
}

Feng Liu ${ }^{\dagger}$, Fei Xue ${ }^{\dagger}$, Yitao $\mathrm{Si}^{\dagger}$, Guijun Chen ${ }^{\dagger}$, Xiangjiu Guan ${ }^{\dagger}$, Kejian Lu${ }^{\dagger}$, Maochang $\mathrm{Liu}^{\dagger,+^{*}}$

International Research Center for Renewable Energy \& State Key Laboratory of Multiphase Flow in Power Engineering, Xi'an Jiaotong University, Xi'an, Shaanxi 710049, P. R. China

'Suzhou Academy of Xi'an Jiaotong University, Suzhou, Jiangsu 215123, P. R. China

*To whom correspondence should be addressed. Email: maochangliu@mail.xjtu.edu.cn (M. Liu);

Phone: +86-(29)-82668296-18; Fax: +86-(29)-82669033. 


\section{Table of contents}

Figure S1. (a) TEM and (b-d) HRTEM images of as-prepared CZS nanotwins.

Figure S2. (a) and (b) TEM images of the common CZS photocatalyst. (c) HRTEM images of the common CZS photocatalyst.

Figure S3. (a) STEM and (b) corresponding elemental mapping images of Zn and P. (c) The EDS spectrum of the obtained CZS-P5.

Figure S4. (a) TEM and (b) HRTEM images of the obtained CZS-P3. (c) STEM image, and (d-g) the corresponding elemental mapping images of (d) Cd, (e) Zn, (f) S, and (g) P of the CZS-P3 photocatalyst.

Figure S5. (a) TEM and (b) HRTEM images of the obtained CZS-P1. (c) STEM image, and (d-g) the corresponding elemental mapping images of (d) Cd, (e) Zn, (f) S, and (g) P of the CZS-P1 photocatalyst.

Figure S6. (a) XRD patterns of the CZS-C, CZS, CZS-P1, CZS-P3 and CZS-P5. (b) Closer investigation over the 24-30 degree and 40-60 degree of the obtained XRD pattern over CZS and CZS-P1-10.

Figure S7. (a) XPS survey spectrum and high resolution XPS spectra of (b) Cd 3d, (c) Zn 2p, and (d) S 2p of CZS, CZS-P1, CZS-P5 and CZS-P7.

Figure S8. (a) Time-coursed photocatalytic $\mathrm{H}_{2}$ evolution activity of CZS-C, CZS, CZS-C-P5 and CZS-P5.

(b) Comparation of photocatalytic $\mathrm{H}_{2}$ evolution rates over CZS-P5 under full acr irradiation and visible light irradiation with different wavelength.

Figure S9. (a) Overview and (b) magnified TEM images of the CZS-P5 after the stability test of 15 hours.

Figure S10. Comparation of the XRD patterns before and after photocatalytic reaction over CZS-P5.

Figure S11. The I-t curves measured by RDE at a rotation rate of $1600 \mathrm{rpm}$ using reaction supernatant, reaction supernatant with $\mathrm{MnO}_{2}$ and pure water as the electrolyte.

Figure S12. Mechanism for the detection of formed $\cdot \mathrm{OH}$ via the reaction between $\cdot \mathrm{OH}$ and terephthalic acid.

Figure S13. Photocatalytic degradation of RhB dye over CZS-P5 with and without BQ.

Figure S14. (a) Uv-vis absorbance spectrum and (b) the Tauc plots of the UV-vis spectra based on $(\mathrm{F}(\mathrm{R}) \mathrm{h} v) 1 / 2$ (Kubelka-Munk function) function of CZS, CZS-P1 and red P.

Figure S15. $(a, b)$ TEM and $(c, d)$ corresponding HRTEM images of the CZS-P5 nanocrystals after photodeposition of Pt.

Table S1. Elemental content of the CZS and CZS-P obtaining from EDS. 
Table S2. Elemental content of the CZS and CZS-P obtaining from ICP-MS.

Table S3. The comparison of CZS-P with representative metal chalcogenide-based photocatalysts toward photocatalytic pure water splitting.
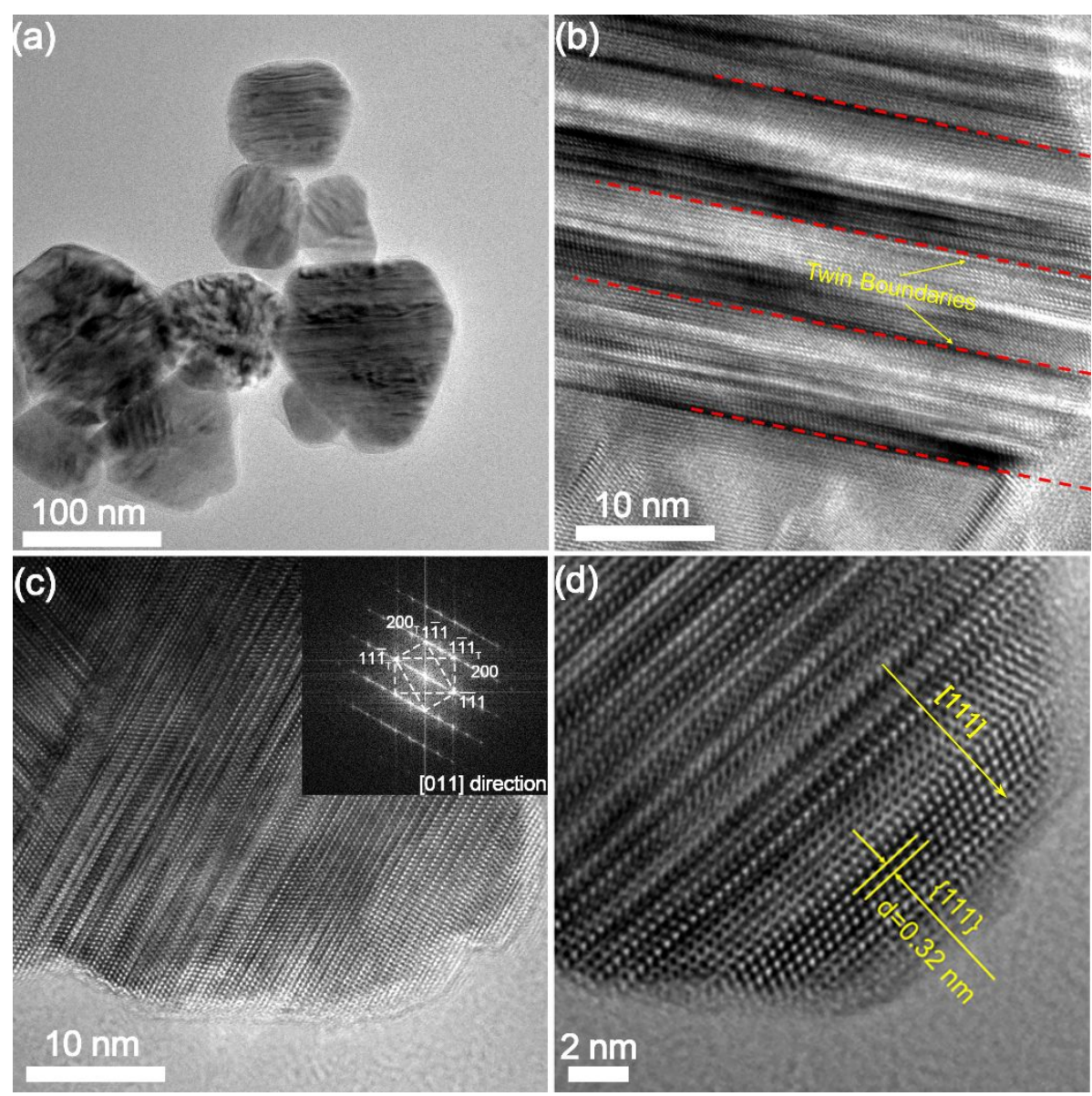

Figure S1. (a) TEM and (b-d) HRTEM images of as-prepared CZS nanotwins. The inset in (c) displays the selected area electron diffraction of CZS sample. 

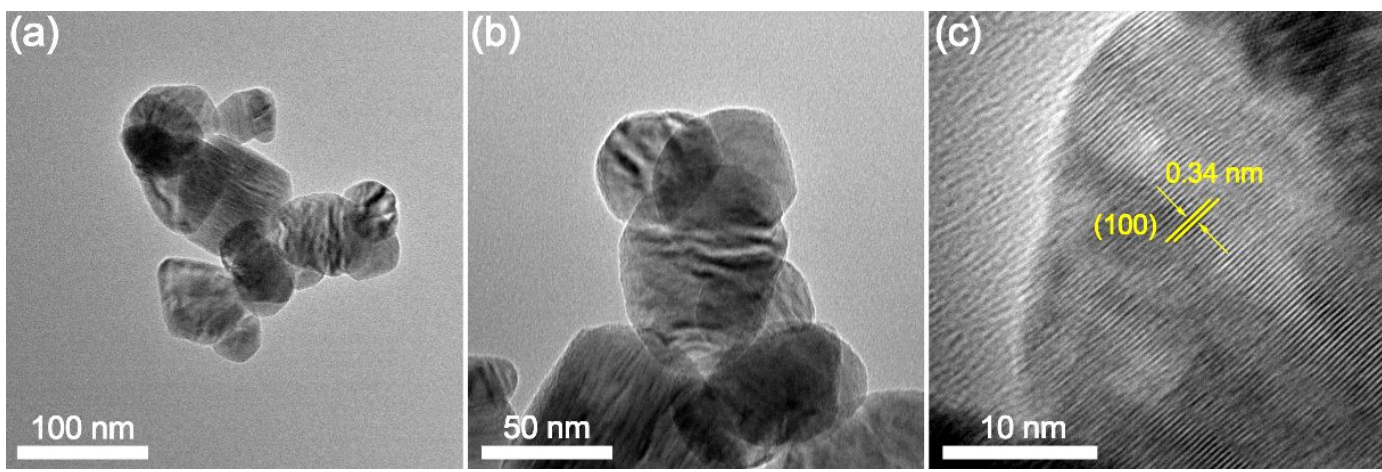

Figure S2. (a) and (b) TEM images of the common CZS photocatalyst. (c) HRTEM images of the common CZS photocatalyst.
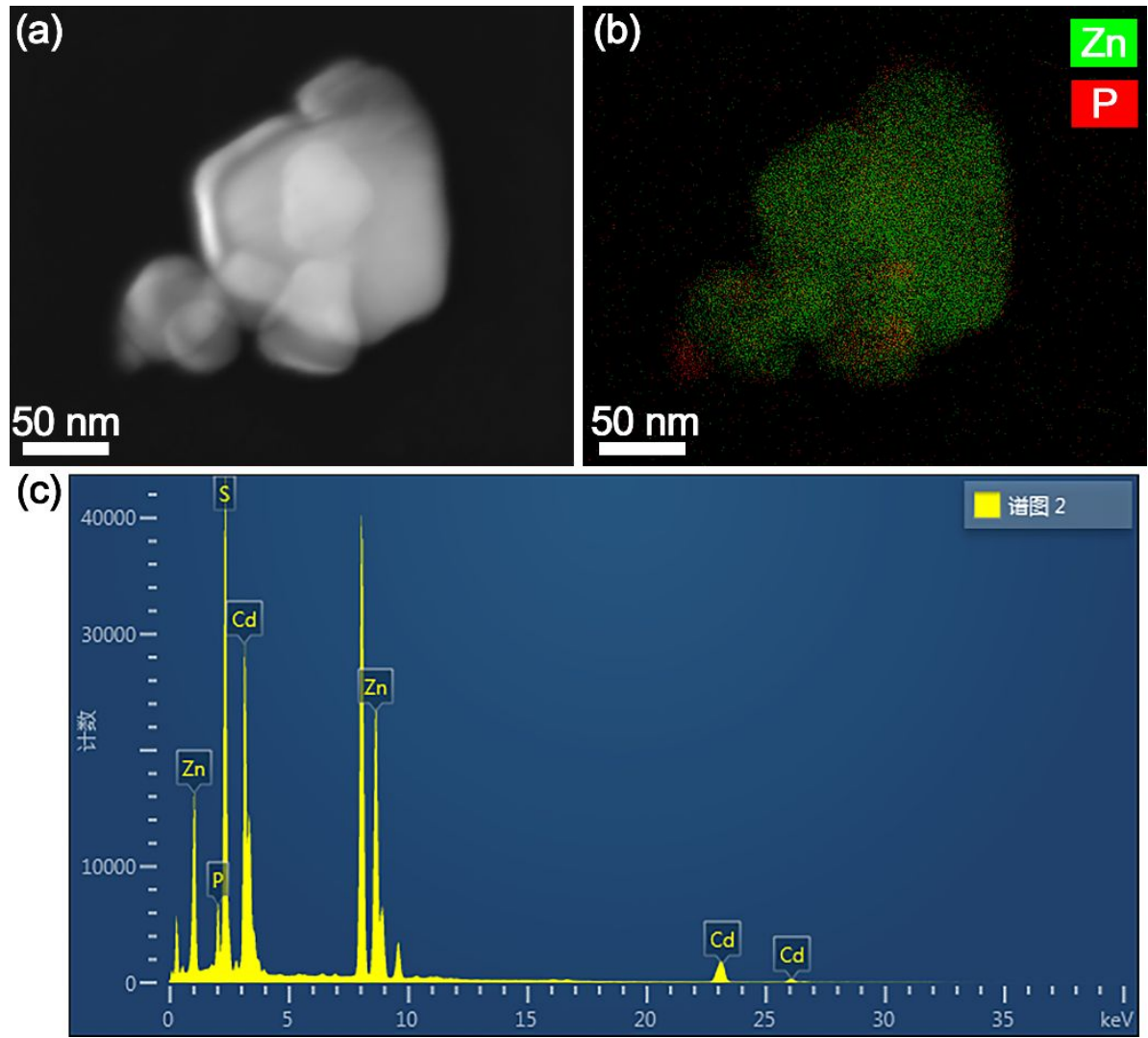

Figure S3. (a) STEM and (b) corresponding elemental mapping images of Zn and P. (c) The EDS spectrum of the obtained CZS-P5. 

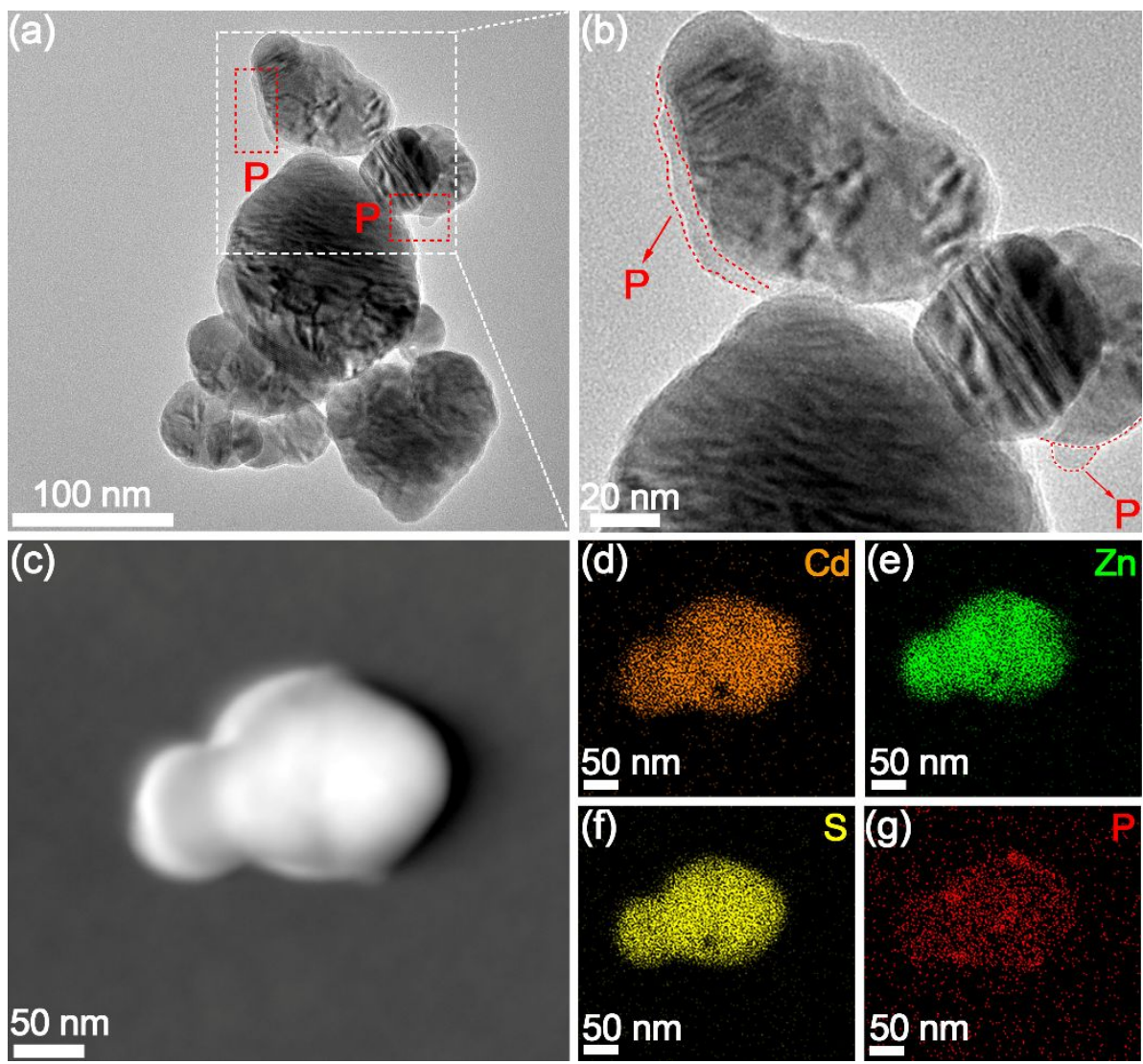

Figure S4. (a) TEM and (b) HRTEM images of the obtained CZS-P3. (c) STEM image, and (d-g) the corresponding elemental mapping images of (d) Cd, (e) Zn, (f) S, and (g) P of the CZS-P3 photocatalyst. As shown in the picture, there is obviously amorphous red phosphorus on the surface of CZS-P3 as we have marked and bulky distribution of $\mathrm{P}$ in the elemental mapping images also demonstrates the existence of red phosphorus. 

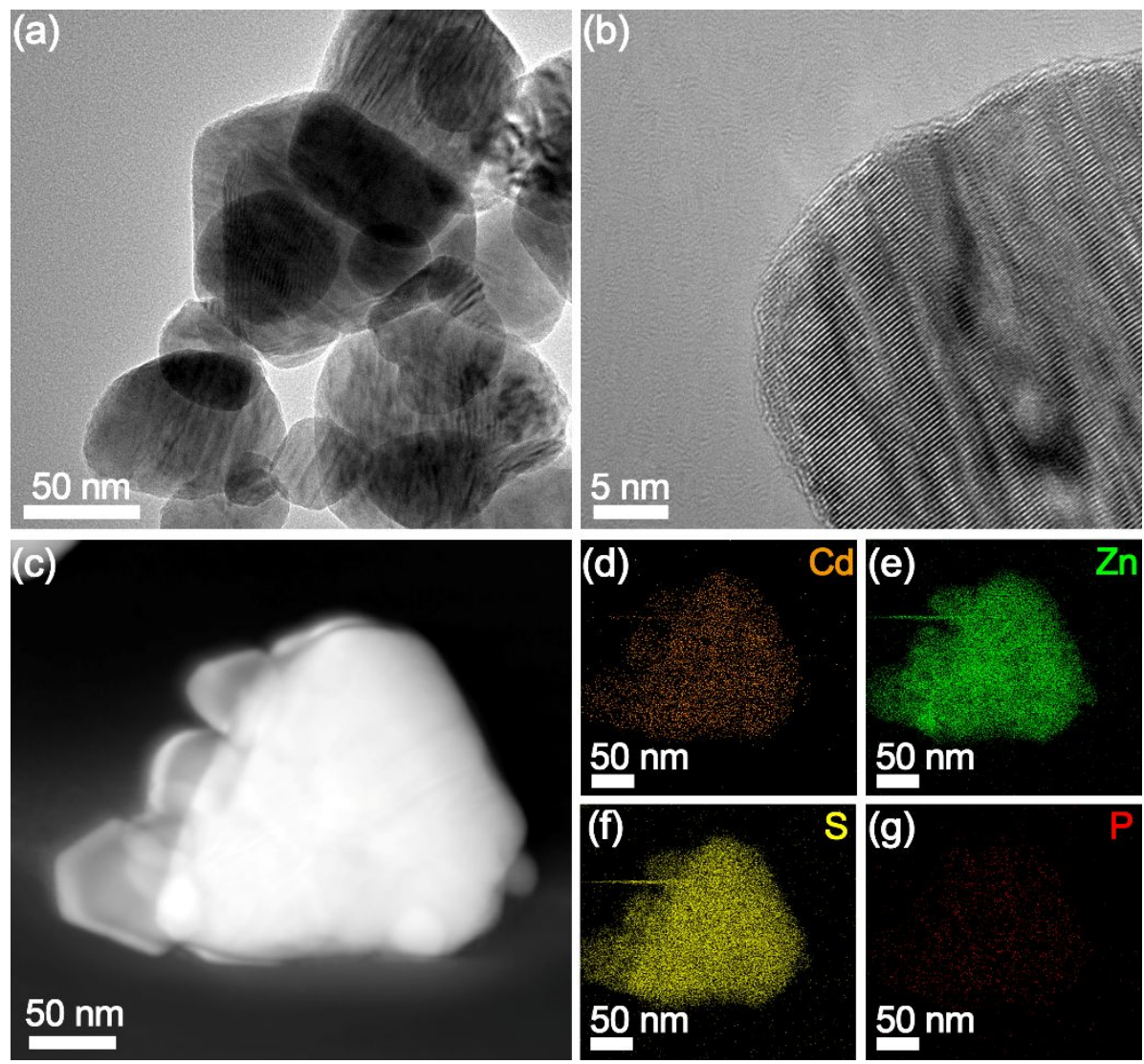

Figure S5. (a) TEM and (b) HRTEM images of the obtained CZS-P1. (c) STEM image, and (d-g) the corresponding elemental mapping images of (d) Cd, (e) Zn, (f) S, and (g) P of the CZS-P1 photocatalyst. There is no red phosphorus on the surface of CZS-P1 and no bulky distribution of $\mathrm{P}$ in the elemental mapping images. 

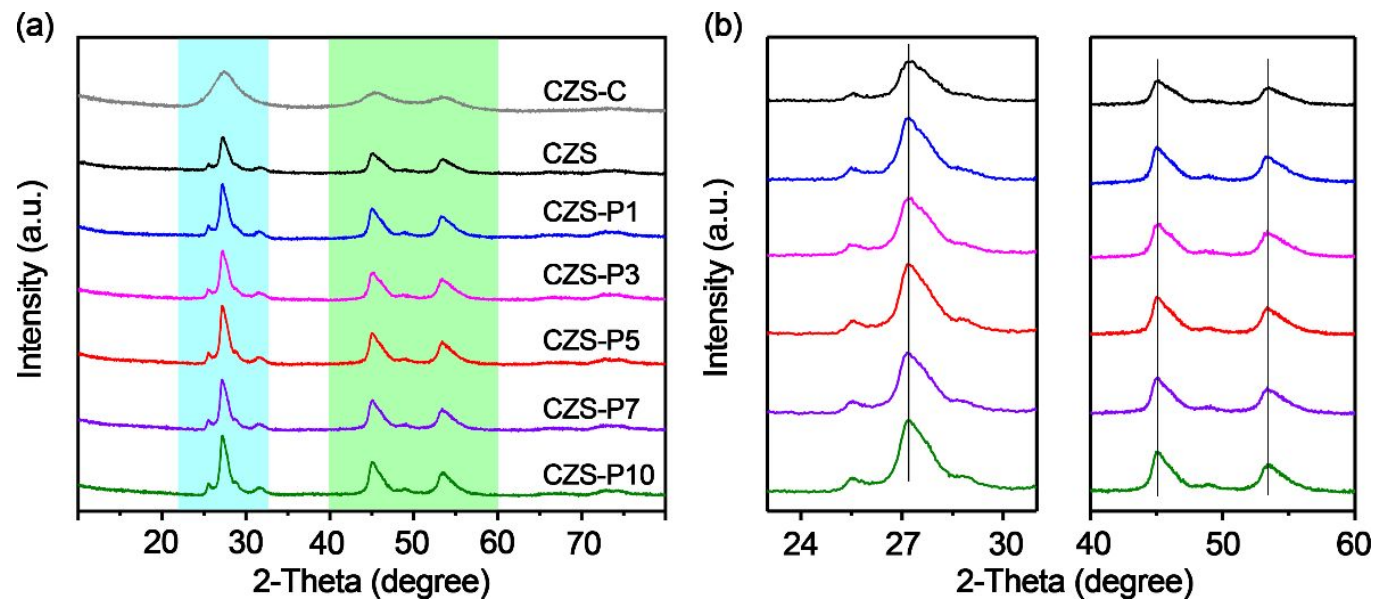

Figure S6. (a) XRD patterns of the CZS-C, CZS, CZS-P1, CZS-P3 and CZS-P5. (b) Closer investigation over the 24-30 degree and 40-60 degree of the obtained XRD pattern over CZS and CZS-P1-10. 

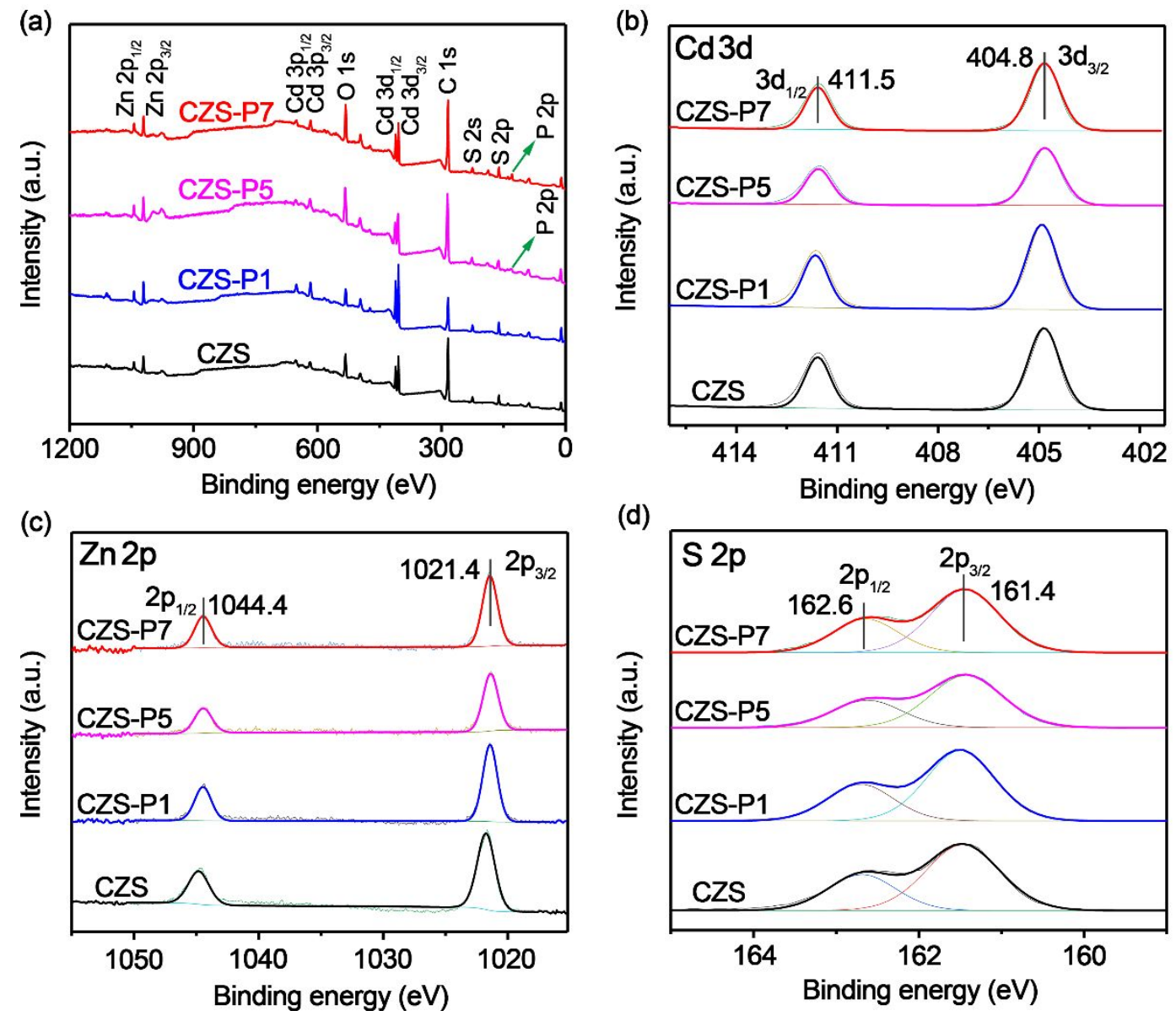

Figure S7. (a) XPS survey spectrum and high resolution XPS spectra of (b) Cd 3d, (c) Zn 2p, and (d) S 2p of CZS, CZS-P1, CZS-P5 and CZS-P7. All the spectra are calibrated with C 1s peak at $284.8 \mathrm{eV}$ of the adventitious carbon. 
(a)

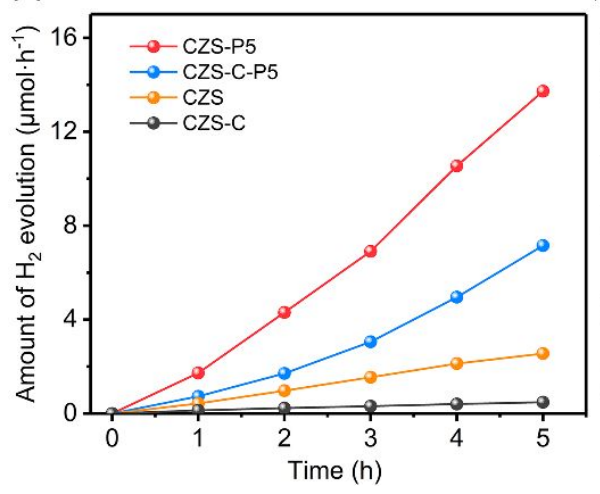

(b)

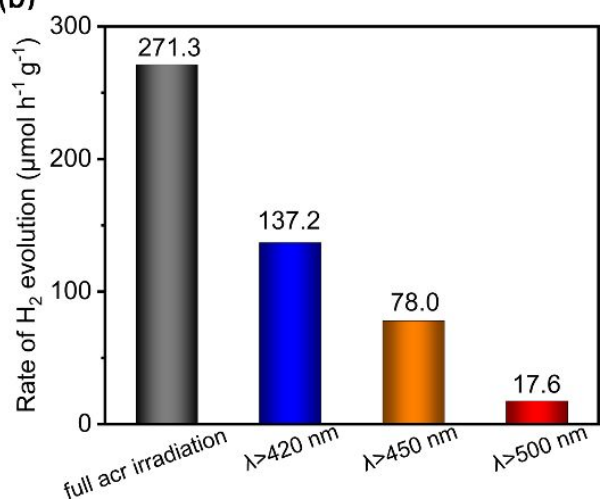

Figure S8. (a) Time-coursed photocatalytic $\mathrm{H}_{2}$ evolution activity of CZS-C, CZS, CZS-C-P5 and CZS-P5. (b) Comparation of photocatalytic $\mathrm{H}_{2}$ evolution rates over CZS-P5 under full acr irradiation and visible light irradiation with different wavelength.
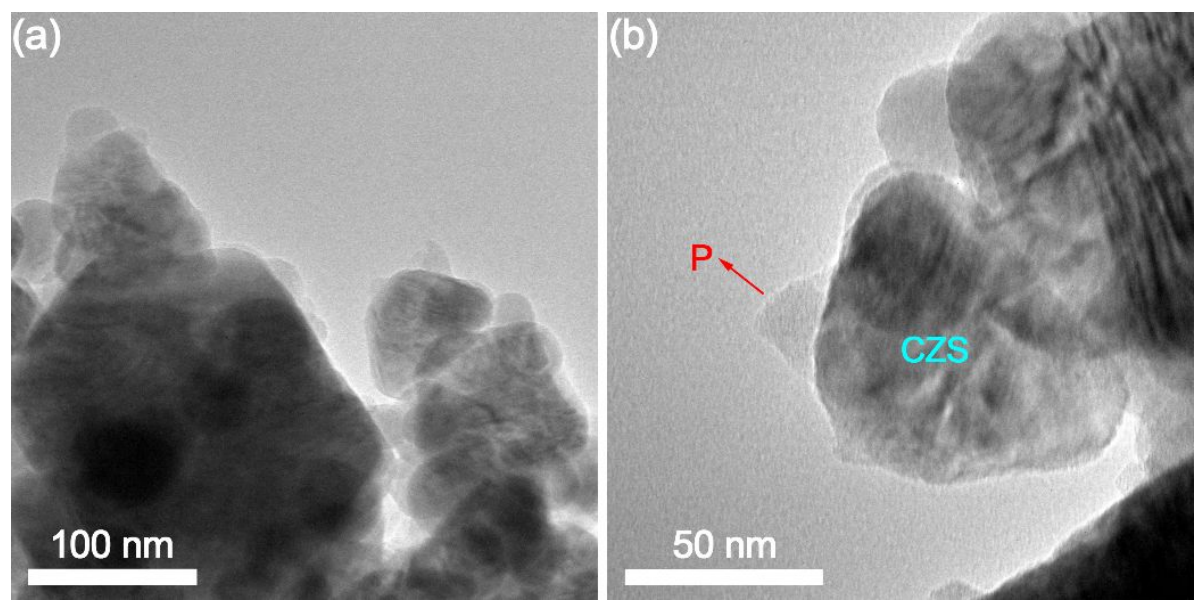

Figure S9. (a) Overview and (b) magnified TEM images of the CZS-P5 after the stability test of 15 hours. 


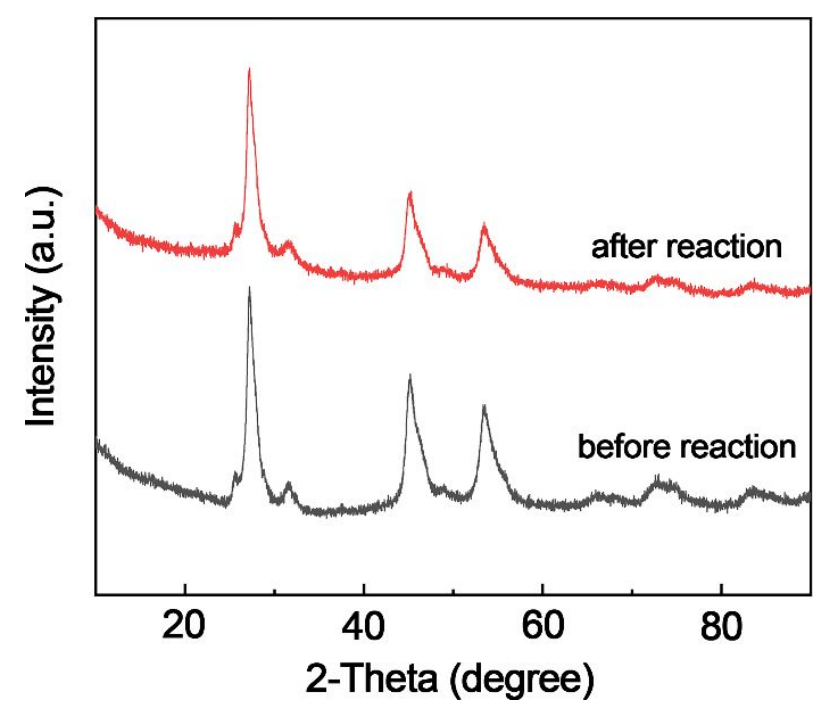

Figure S10. Comparation of the XRD patterns before and after photocatalytic reaction over CZS-P5.

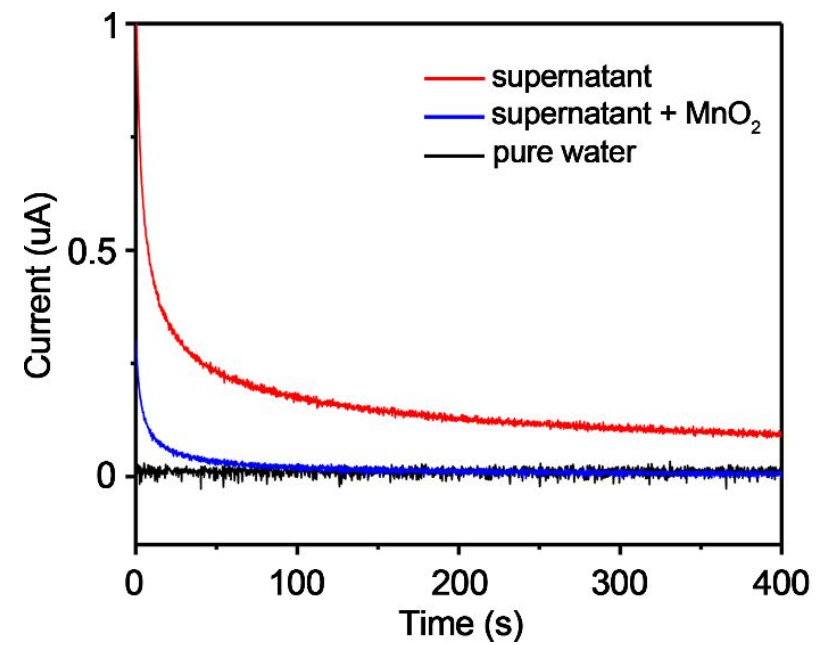

Figure S11. The I-t curves measured by RDE at a rotation rate of $1600 \mathrm{rpm}$ using reaction supernatant, reaction supernatant with $\mathrm{MnO}_{2}$ and pure water as the electrolyte.<smiles>O=C(O)c1ccc(C(=O)O)cc1</smiles>

Terephthalic acid Without fluorescence<smiles>O=C(O)c1ccc(C(=O)O)c(O)c1</smiles>

2-hydroxyterephthalic acid

\section{With fluorescence}

Figure S12. Mechanism for the detection of formed $\cdot \mathrm{OH}$ via the reaction between $\cdot \mathrm{OH}$ and terephthalic acid. 


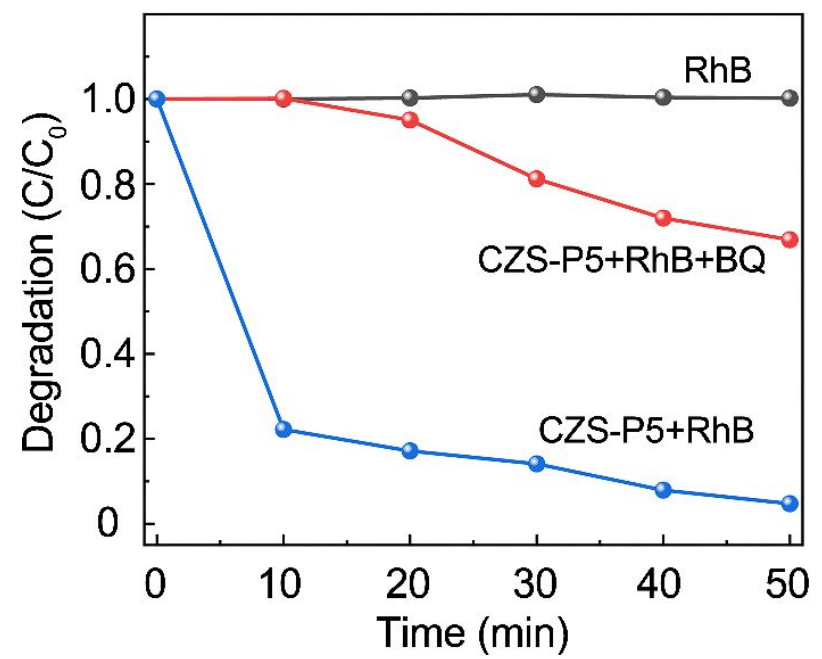

Figure S13. Photocatalytic degradation of RhB dye over CZS-P5 with and without BQ.
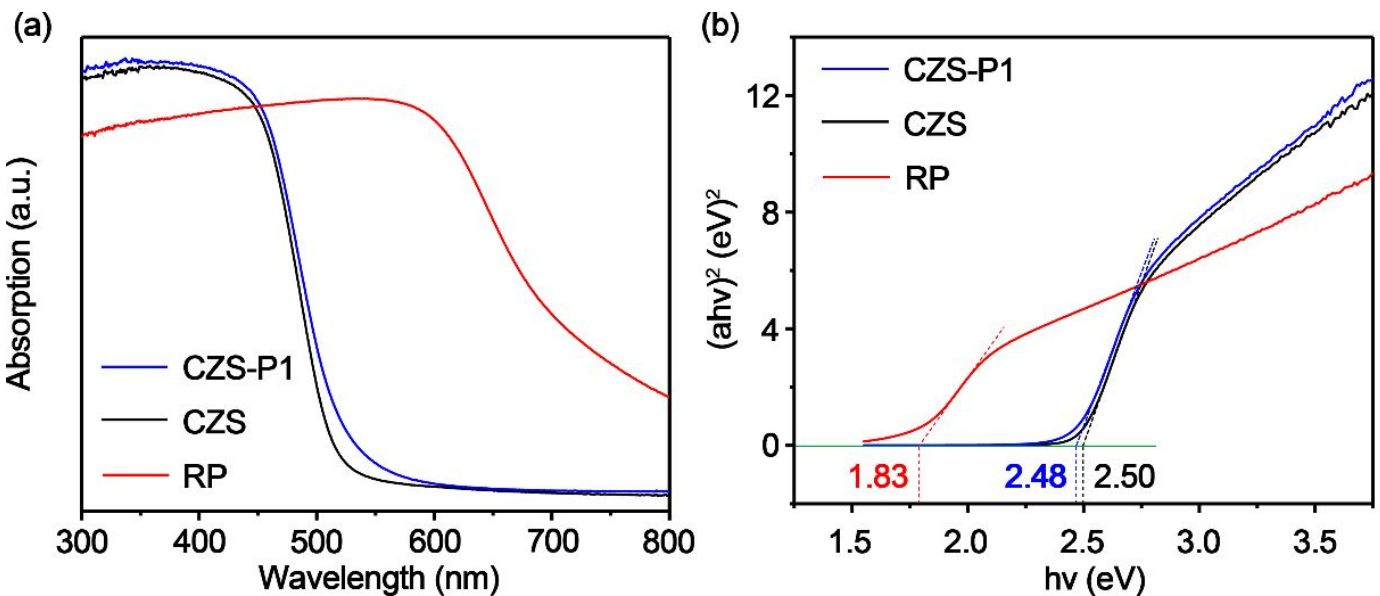

Figure S14. (a) Uv-vis absorbance spectrum and (b) the Tauc plots of the UV-vis spectra based on $(\mathrm{F}(\mathrm{R}) \mathrm{h} v) 1 / 2$ (Kubelka-Munk function) function of CZS, CZS-P1 and red P. The band gap energy (Eg) of these photocatalysts are reveled in (b). 

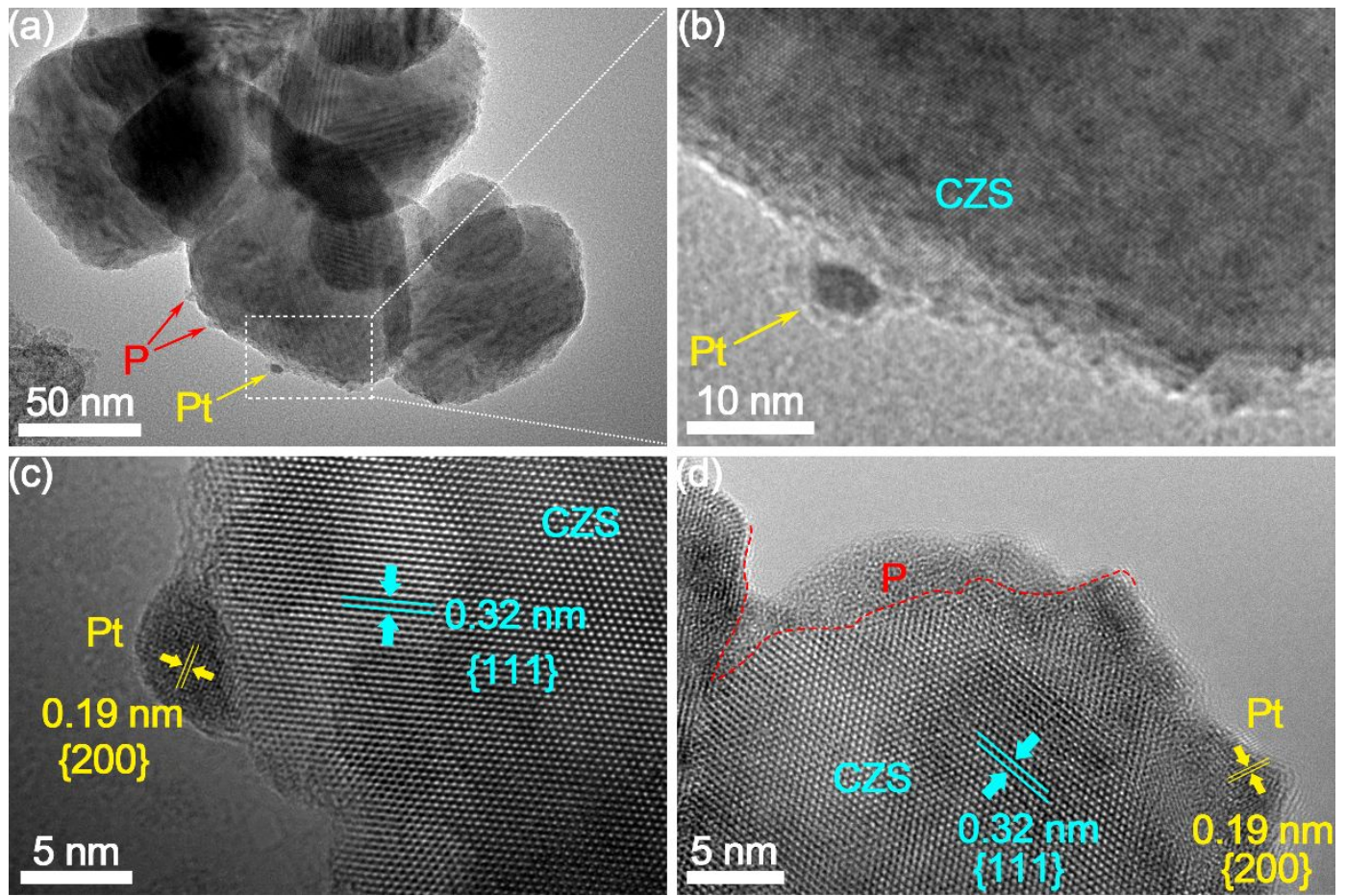

Figure S15. (a,b) TEM and (c,d) corresponding HRTEM images of the CZS-P5 nanocrystals after photodeposition of Pt. The corresponding lattice fringes distance clearly reveal that the Pt nanoparticles are deposited on the surface of CZS rather than red $\mathrm{P}$. 
Table S1. Elemental content of the CZS and CZS-P obtaining from EDS.

\begin{tabular}{ccccc}
\hline Photocatalyst & $\begin{array}{c}\text { Cd content } \\
\text { atomic (\%) }\end{array}$ & $\begin{array}{c}\text { Zn content } \\
\text { atomic (\%) }\end{array}$ & $\begin{array}{c}\text { S content } \\
\text { atomic (\%) }\end{array}$ & $\begin{array}{c}\text { P content } \\
\text { atomic (\%) }\end{array}$ \\
\hline CZS & 21.93 & 23.95 & 54.11 & - \\
CZS-P1 & 22.49 & 24.66 & 52.25 & 0.60 \\
CZS-P3 & 19.77 & 21.78 & 53.33 & 5.13 \\
CZS-P5 & 22.17 & 22.61 & 48.41 & 6.82 \\
\hline
\end{tabular}

Table S2. Elemental content of the CZS and CZS-P obtaining from ICP-MS.

\begin{tabular}{cccc}
\hline Photocatalyst & $\begin{array}{c}\text { Cd content } \\
\text { atomic (\%) }\end{array}$ & $\begin{array}{c}\text { Zn content } \\
\text { atomic (\%) }\end{array}$ & $\begin{array}{c}\text { P content } \\
\text { atomic (\%) }\end{array}$ \\
\hline CZS & 3.32 & 3.15 & - \\
CZS-P1 & 3.57 & 3.73 & 0.10 \\
CZS-P3 & 3.58 & 3.22 & 1.02 \\
CZS-P5 & 3.35 & 3.49 & 1.19 \\
\hline
\end{tabular}

Table S3. The comparison of CZS-P with representative metal chalcogenide-based photocatalysts toward photocatalytic pure water splitting.

\begin{tabular}{|c|c|c|c|c|}
\hline \multirow[b]{2}{*}{ Photocatalyst } & \multirow[b]{2}{*}{ Cocatalyst } & \multicolumn{3}{|c|}{$\mathrm{H}_{2}$ evolution rate } \\
\hline & & AQY (\%) & $\left(\mu \mathrm{mol} \mathrm{h} \mathbf{h}^{-1} \mathrm{~g}^{-1}\right)$ & Ref. (Year) \\
\hline $\mathrm{CdS} @ \mathrm{Al}_{2} \mathrm{O}_{3}$ & $\mathrm{Pt}$ & $0.11(430 \mathrm{~nm})$ & $62.1(\lambda \geq 420 \mathrm{~nm})$ & $1(2018)$ \\
\hline $\mathrm{TiO}_{2} / \mathrm{CdS}$ & $\mathrm{Pt}$ & $0.17(430 \mathrm{~nm})$ & $3.074(\lambda \geq 420 \mathrm{~nm})$ & $2(2017)$ \\
\hline $\mathrm{Zn}_{0.8} \mathrm{Cd}_{0.2} \mathrm{~S}$ & $\mathrm{NiO}$ & $0.66(430 \mathrm{~nm})$ & $99.9(\lambda \geq 420 \mathrm{~nm})$ & 3 (2019) \\
\hline CdS-2017 & $\begin{array}{c}\text { Carbon } \\
\text { dots }\end{array}$ & None & $51(\lambda \geq 420 \mathrm{~nm})$ & $4(2017)$ \\
\hline $\mathrm{MnS} / \mathrm{CoS}_{2}$ & $\begin{array}{c}\text { Carbon } \\
\text { dots }\end{array}$ & None & $4.21(\lambda \geq 420 \mathrm{~nm})$ & $5(2018)$ \\
\hline $\mathrm{P}-\mathrm{Zn}_{\mathrm{x}} \mathrm{Cd}_{1-\mathrm{x}} \mathrm{S}$ & None & 0.12 & 419 (LED light) & $6(2018)$ \\
\hline $\mathrm{CdS} / \mathrm{g}-\mathrm{C}_{3} \mathrm{~N}_{4}$ & $\mathrm{Ni}_{2} \mathrm{P}$ & $0.18(420 \mathrm{~nm})$ & $15.56(\lambda \geq 420 \mathrm{~nm})$ & 7 (2019) \\
\hline $\mathrm{P}-\mathrm{Zn}_{0.5} \mathrm{Cd}_{0.5} \mathrm{~S}_{1-\mathrm{x}}$ & None & $0.15(420 \mathrm{~nm})$ & $32.33(\lambda \geq 420 \mathrm{~nm})$ & $8(2020)$ \\
\hline
\end{tabular}




$\mathrm{Cd}_{0.5} \mathrm{Zn}_{0.5} \mathrm{~S}-\mathrm{P} \quad$ None $\quad 0.32(420 \mathrm{~nm}) \quad 137.2(\lambda \geq 420 \mathrm{~nm}) \quad$ This work

\section{References}

(1) Ning, X.; Zhen, W.; Wu, Y.; Lu, G. Inhibition of CdS Photocorrosion by $\mathrm{Al}_{2} \mathrm{O}_{3}$ Shell for Highly Stable Photocatalytic Overall Water Splitting under Visible Light Irradiation. Appl. Catal. B Environ. 2018, 226 (September 2017), 373-383.

(2) Ning, X.; Li, J.; Yang, B.; Zhen, W.; Li, Z.; Tian, B.; Lu, G. Inhibition of Photocorrosion of CdS via Assembling with Thin Film $\mathrm{TiO}_{2}$ and Removing Formed Oxygen by Artificial Gill for Visible Light Overall Water Splitting. Appl. Catal. B Environ. 2017, 212, 129-139.

(3) Ning, X.; Zhen, W.; Zhang, X.; Lu, G. Assembly of Ultra-Thin NiO Layer Over $\mathrm{Zn}_{1-\mathrm{x}} \mathrm{Cd}_{\mathrm{x}} \mathrm{S}$ for Stable Visible-Light Photocatalytic Overall Water Splitting. ChemSusChem 2019, 12, 1410-1420.

(4) Zhu, C.; Liu, C.; Zhou, Y.; Fu, Y.; Guo, S.; Li, H.; Zhao, S.; Huang, H.; Liu, Y.; Kang, Z. Carbon Dots Enhance the Stability of CdS for Visible-Light-Driven Overall Water Splitting. Appl. Catal. B Environ. 2017, 216, 114-121.

(5) Fu, Y.; Zhu, C.; Liu, C.; Zhang, M.; Wang, H.; Shi, W.; Huang, H.; Liu, Y.; Kang, Z. CoMn-S/CDs Nanocomposite for Effective Long Wavelength Visible-Light-Driven Photocatalytic Water Splitting. Appl. Catal. B Environ. 2018, 226, 295-302.

(6) Ye, H. F.; Shi, R.; Yang, X.; Fu, W. F.; Chen, Y. P-Doped $Z_{\mathrm{x}} \mathrm{Cd}_{1-\mathrm{x}} \mathrm{S}$ Solid Solutions as Photocatalysts for Hydrogen Evolution from Water Splitting Coupled with Photocatalytic Oxidation of 5-Hydroxymethylfurfural. Appl. Catal. B Environ. 2018, 233, 70-79.

(7) He, H.; Cao, J.; Guo, M.; Lin, H.; Zhang, J.; Chen, Y.; Chen, S. Distinctive Ternary $\mathrm{CdS} / \mathrm{Ni}_{2} \mathrm{P} / \mathrm{g}-\mathrm{C}_{3} \mathrm{~N}_{4}$ Composite for Overall Water Splitting: $\mathrm{Ni}_{2} \mathrm{P}$ Accelerating Separation of Photocarriers. Appl. Catal. B Environ. 2019, 249, 246-256.

(8) Ng, B.-J.; Putri, L. K.; Kong, X. Y.; Pasbakhsh, P.; Chai, S.-P. Overall Pure Water Splitting Using One-Dimensional P-Doped Twinned $\mathrm{Zn}_{0.5} \mathrm{Cd}_{0.5} \mathrm{~S}_{1-\mathrm{x}}$ Nanorods via Synergetic Combination of Long-Range Ordered Homojunctions and Interstitial $\mathrm{S}$ Vacancies with Prolonged Carrier Lifetime. Appl. Catal. B Environ. 2020, 262, 118309. 